\title{
Use of high-density tiling microarrays to identify mutations globally and elucidate mechanisms of drug resistance in Plasmodium falciparum
}

\author{
Neekesh V Dharia*, Amar Bir Singh Sidhu ${ }^{\dagger \neq}$, María Belén Cassera§, \\ Scott J Westenberger*, Selina ER Bopp*, Rich T Eastman ${ }^{\dagger}$, David Plouffeף,

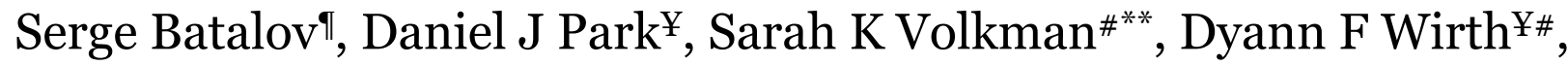 \\ Yingyao Zhou", David A Fidock ${ }^{\dagger \dagger}$ and Elizabeth A Winzeler*ף
}

\begin{abstract}
Addresses: * Department of Cell Biology, ICND 202, The Scripps Research Institute, North Torrey Pines Road, La Jolla, CA 92037, USA. ${ }^{\dagger}$ Department of Microbiology, Columbia University College of Physicians and Surgeons, West 186th Street, New York, NY 10032, USA. ${ }^{\ddagger}$ The Broad Institute of MIT and Harvard, Cambridge Center, Cambridge, MA 02142, USA. §Department of Biochemistry, Albert Einstein College of Medicine at Yeshiva University, Morris Park Avenue, Bronx, NY 10461, USA. "Genomics Institute of the Novartis Research Foundation, John Jay Hopkins Drive, San Diego, CA 92121, USA. ${ }^{¥}$ The Broad Institute of MIT and Harvard, Cambridge Center, Cambridge, MA 02142, USA. \#Department of Immunology and Infectious Diseases, Harvard School of Public Health, Huntington Avenue, Boston, MA 02115, USA. **School for Health Studies, Simmons College, The Fenway, Boston, MA 02115, USA. ${ }^{\dagger+}$ Department of Medicine, Columbia University College of Physicians and Surgeons, West 186th Street, New York, NY 10032, USA.
\end{abstract}

Correspondence: Elizabeth A Winzeler. Email: winzeler@scripps.edu

Published: 13 February 2009

Genome Biology 2009, 10:R2I (doi:10.1 I86/gb-2009-10-2-r2I)

The electronic version of this article is the complete one and can be found online at http://genomebiology.com/2009//0/2/R2I
Received: 20 September 2008

Revised: 5 January 2009

Accepted: 13 February 2009

(C) 2009 Dharia et al.; licensee BioMed Central Ltd.

This is an open access article distributed under the terms of the Creative Commons Attribution License (http://creativecommons.org/licenses/by/2.0), which permits unrestricted use, distribution, and reproduction in any medium, provided the original work is properly cited.

\begin{abstract}
Background: The identification of genetic changes that confer drug resistance or other phenotypic changes in pathogens can help optimize treatment strategies, support the development of new therapeutic agents, and provide information about the likely function of genes. Elucidating mechanisms of phenotypic drug resistance can also assist in identifying the mode of action of uncharacterized but potent antimalarial compounds identified in high-throughput chemical screening campaigns against Plasmodium falciparum.
\end{abstract}

Results: Here we show that tiling microarrays can detect de novo a large proportion of the genetic changes that differentiate one genome from another. We show that we detect most single nucleotide polymorphisms or small insertion deletion events and all known copy number variations that distinguish three laboratory isolates using readily accessible methods. We used the approach to discover mutations that occur during the selection process after transfection. We also elucidated a mechanism by which parasites acquire resistance to the antimalarial fosmidomycin, which targets the parasite isoprenoid synthesis pathway. Our microarray-based approach allowed us to attribute in vitro derived fosmidomycin resistance to a copy number variation event in the pfdxr gene, which enables the parasite to overcome fosmidomycin-mediated inhibition of isoprenoid biosynthesis.

Conclusions: We show that newly emerged single nucleotide polymorphisms can readily be detected and that malaria parasites can rapidly acquire gene amplifications in response to in vitro drug pressure. The ability to define comprehensively genetic variability in $P$. falciparum with a single overnight hybridization creates new opportunities to study parasite evolution and improve the treatment and control of malaria. 


\section{Background}

With many complete eukaryotic genomes and draft eukaryotic sequencing projects deposited in the National Center for Biotechnology Information database, attention is shifting to discovering genomic diversity and associating this genetic variation with defined phenotypes. This is of particular interest with the human malarial parasite Plasmodium falciparum, whose extensive genetic variability and sexual recombination facilitates the emergence and spread of drug resistance $[1,2]$, resulting in treatment failure for many of the licensed antimalarial agents [3,4]. Identifying the genetic changes that are involved in drug resistance or other phenotypic changes can help with the development of effective therapies, improve understanding of parasite biology and gene function, and assist in elucidating the mode of action of uncharacterized chemical compounds that exhibit antimalarial activity in high-throughput cellular screening campaigns [5-7]. Traditional genetic methods have been used to discover such genetic changes but with much difficulty, time, and cost for the experimentally intractable $P$. falciparum.

Traditional forward genetic methods that have been used to discover Plasmodium genes involved in drug resistance include genetic crosses and analysis of linkage patterns of sexual assortment that occur naturally during parasite transmission from mammal to insect. For example, the primary genetic determinant of chloroquine drug resistance in $P$. falciparum was identified through a costly genetic cross involving chimpanzees [8,9]. Allelic replacement experiments confirmed that resistance was mediated by point mutations in the chloroquine resistance transporter ( $p f c r t$, MAL7P1.27) [10]. Crosses can also be performed at a significantly reduced cost using rodent malaria models, but the mechanism of drug resistance in these systems may not extend to human malaria [11]. In certain instances, linkage disequilibrium studies of sensitive and resistant field isolates, using single nucleotide polymorphisms (SNPs) reported by the recent sequencing projects [12-14], can also uncover genetic determinants of resistance. Indeed, recent analysis of such data has identified selective sweeps associated with chloroquine and antifolate drug resistance $[12,15]$.

An alternative reverse genetic approach leverages knowledge from other systems to predict the candidate genes that might be involved in antimalarial drug resistance. For instance, membrane transporters encoded by multidrug resistance $(m d r)$ genes can contribute to drug resistance in other organisms. In the case of $P$. falciparum, amplification of the $p$ fmdr 1 gene (PFE115Ow) leads to mefloquine resistance [16,17], and point mutations in this gene modulate in vitro susceptibility to multiple antimalarial agents $[1,18,19]$. SNPs in the dihydrofolate reductase-thymidylate synthase gene ( $p f d h f r$-ts, PFDo830w) confer resistance to antifolate drugs $[1,18,20]$, and a candidate gene approach has been used to successfully correlate in vitro derived resistance to the macrolide azithromycin with a point mutation in a ribosomal protein that is part of the apicoplast translation machinery [21]. These candidate gene approaches, however, have limited predictive value with drugs that are specific to malarial parasites and have unknown modes of action.

Not withstanding some earlier successes with classical genetic approaches, technological advances in genomics research are beginning to afford unprecedented power in genome-wide discovery of mutations, thus facilitating highthroughput approaches for discovering genes that are involved in drug resistance. Particularly in laboratoryadapted isogenic isolates, genomic methods are making it possible to differentiate between genetic changes associated with resistance and random mutations. Genome re-sequencing has proven especially useful for SNP detection; however, its primary limitation is in not being able to detect copy number variations (CNVs), which are likely to be a common response to drug pressure. Alternative microarray-based approaches have been used to discover a novel amplification event surrounding GTP cyclohydrolase I (pfgch1, PFL1155w) that may be important for antifolate drug resistance [22,23]. Microarray approaches can rapidly identify variable genomic regions and assess CNVs at relatively low cost, providing a distinct advantage over conventional sequencing approaches.

Here we describe the production of a custom high-density tiling 25mer oligonucleotide microarray, based on the 3D7 reference isolate, and the development of analytical tools for the genome-wide identification of mutations in laboratory and clinical isolates of $P$. falciparum. This enabled us to identify more than $90 \%$ of reported SNPs in Dd2 with respect to $3 \mathrm{D} 7$, and the precise mapping of amplification events surrounding pfmdr1 and pfgch1, all in a fast, single hybridization experiment. We illustrate the utility of this approach by reporting evidence of a molecular and biochemical basis of parasite resistance to fosmidomycin: amplification of the gene encoding the putative target $P$. falciparum 1-deoxy-D-xylulose $5^{-}$ phosphate reductoisomerase ( $p f d x r, \mathrm{PF} 14 \_0641$ ) in parasite lines selected for decreased fosmidomycin susceptibility in vitro.

\section{Results}

\section{Microarray design}

One goal of this study was to determine whether we could use hybridization methods to detect SNPs and CNVs globally in the $P$. falciparum genome and to implement a robust and user-friendly software package to analyze these data. We first constructed a custom high-density microarray containing over 4.8 million probes at $5 \mu \mathrm{m}$ feature size to the sequenced 3D7 isolate [24]. The microarray covers approximately $90 \%$ of coding regions, which comprise $53 \%$ of the genome, and $60 \%$ of noncoding regions, and it is only limited by the high AT content of the $P$. falciparum genome [24]. Unlike the yeast tiling microarray [25], our microarray contains only perfect match probes, and thus we were able to tile through 
the genome with overlapping probes of alternating strandedness averaging 25 nucleotides in length with a base pair spacing of two to three nucleotides. Thus, each nucleotide in the coding genome could be probed from 9 to 13 times on the microarray. This probe density allows for detection of polymorphisms within a distance of several nucleotides, and the density is much greater than our previous $P$. falciparum microarray that had 327,989 non-overlapping 25 mer oligonucleotide probes spaced approximately every 50 base pairs [22] or the microarray, also used for SNP and CNV detection, designed at The Sanger Institute that has about 2.5 million probes [26].

To validate our microarray-based approach, genomic DNA preparations from the culture-adapted $\mathrm{Dd}_{2}, \mathrm{HB}_{3}$, and $3 \mathrm{D}_{7}$ parasite lines were labeled and hybridized to the microarray. We used these lines as references because of the availability of high-quality sequence data generated by traditional sequencing methods. Following hybridization, the arrays were washed and scanned, and the microarray data normalized to a baseline synthetic array using the nonlinear piece-wise running median line for invariant probes [27]. This synthetic array was constructed by taking the mean for each probe across all arrays for analysis. This method of normalization was chosen over quantile normalization [27], a popular method for gene expression analysis, because the reference isolate and other isolates were expected to have different distributions of probe intensities as a consequence of their natural genetic variation.

\section{Gene amplification and deletion events}

We next sought to determine whether we could detect known CNVs and deletions using our microarray-based approach, in view of the key role that CNVs can play in the phenotypes of diverse organisms, including malarial parasites. For this analysis, we used only probes that mapped to only one location in the $3 \mathrm{D} 7$ reference genome ( $87.7 \%$ of total probes). The data were subject to two different analyses. First, we systematically detected gene deletion events using a match-only integral distribution (MOID) algorithm [28], similar to one previously used in our laboratory to detect CNVs with the previous generation of microarrays [22]. For this analysis, we modeled the background hybridization by using Affymetrix eukaryotic background control probes. This required at least ten unique probes per gene, and thus we excluded 95 highly variable subtelomeric genes (Additional data file 1). We considered one of two criteria to designate a gene as absent: the probe hybridization results fell within the distribution of background probes with a $P$ value cutoff of $1 \times 10^{-2}$; or the gene had less than a 2.5-fold increase in intensity at the 70th percentile with respect to background. Using the MOID algorithm, we identified a total of 5,461 genes with ten or more probes in $3 \mathrm{D} 7$ as present, indicating a low false-positive rate. We also confirmed that a group of six subtelomeric cytoadherence genes on chromosome 2 were deleted in Dd2 com- pared with $3 \mathrm{D} 7$, in accordance with the findings of a previous report [22] (Additional data file 1).

Second, we detected amplification events by determining the $\log _{2}$ ratio of unique probes in the isolate compared with the reference isolate. Each chromosome was scanned with sliding windows of 1,000 probes, and a z-test was performed on each window to determine whether the probes had a $\log _{2}$ ratio greater than zero. We were able to identify known amplification events in Dd2 surrounding pfmdr1 on chromosome 5 and pfgch1 on chromosome 12 [22] using a z-value cutoff of 18 , which was used as a cutoff for the analysis below (Additional data file 2). Quantitative PCR had earlier determined that this isolate harbored 3-4 copies of pfmdr1 [29,30], and quantitative RT-PCR of our freshly cloned Dd2 line indicated the presence of three copies (data not shown). With our method, we did not detect a threefold increase in intensity for this region. Instead we saw a 1.6-fold intensity increase, with a $\log _{2}$ ratio of approximately 0.7 (Figure 1). Hybridizationbased methods may not be linear with respect to copy number detection because of probe or scanner signal saturation. Linearity could possibly be achieved by shortening the hybridization times or by performing titrations. However, it would probably be more efficient to perform quantitative PCR than to collect whole-genome data. The goal of our study was to discover CNVs and not to extensively characterize them.

With a large number of probes to both coding and intergenic regions, we predicted that our method would allow us to delineate the breakpoints of amplification events with high accuracy, when comparing isolate genomic hybridizations with a reference isolate. To determine whether we could precisely detect the previously determined boundaries of amplification events, we scanned through the regions surrounding the amplifications and performed a paired $t$-test comparing the probe intensities on either side of each position on a chromosome with the window size dependent on the size of the amplification event. The position with the best $t$-statistic was considered to be the breakpoint of the amplification event, and reported ranges included positions with a $t$-statistic greater than $90 \%$ of the peak.

PCR analysis of the pfmdr1 amplification event in Dd2 previously estimated the breakpoints to be 888,335 on the 5 ' end and 970,240 on the 3 ' end [31]. Microarray-based mapping localized the amplification breakpoints at approximately $888,543(888,393$ to 888,689$)$ and $970,202(969,734$ to $970,348$ ) with a 2 kilobase $(\mathrm{kb})$ window size (Figure 1$)$. This amplification event could not be as precisely mapped because the breakpoints occur in intergenic tracts of monomeric A or $\mathrm{T}$; nevertheless, we were still able to identify the region to within a few hundred bases. Our method also identified the GTP cyclohydrolase I amplification event in Dd2, which included three genes, namely PFL1145w, PFL1150c, and PFL1155w (pfgch1), confirming our initial report [22]. With our high-density microarray, we detected the breakpoints of 


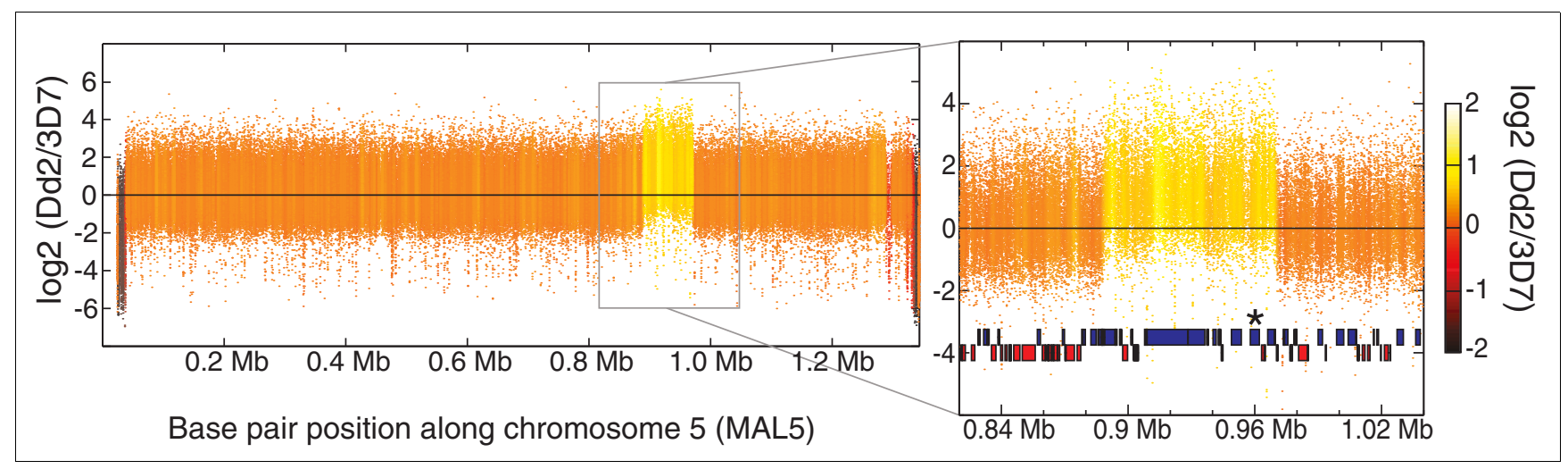

Figure I

Amplification event surrounding pfmdrl on chromosome 5 in Dd2. The $\log _{2}$ ratio of the intensity of each unique probe in Dd2 was divided by the intensity of each unique probe in 3D7 to generate the plot. The probe log ratios were colored by the moving average over a 500 base pair window as indicated in the color bar. A normal distribution around zero was expected if the copy number was the same. The ends of chromosome 5 are highly polymorphic subtelomeric regions and thus showed much lower probe intensity in Dd2 when compared with the reference 3D7. The amplification containing I4 genes including pfmdrl (marked with an asterisk) is enlarged in the inset. Mb, megabases.

this amplification as $971,195(971,185$ to 971,218$)$ and 976,476 $(976,448$ to 976,525$)$ using a 500 base pair window size. In HB3, the amplification event surrounding pfgch1 was much larger, about $161 \mathrm{~kb}$, with breakpoints at 942,424 (942,144 to 942,717) and $1,103,325(1,103,153$ to $1,103,624)$ when scanned with a $4 \mathrm{~kb}$ window size. Our initial report indicated this amplification event contained 39 genes (PFL1125W to PFL1315w) [22], but our higher resolution microarray identified the breakpoints within the coding regions of PFL1125W and PFL1315w, suggesting a full-length CNV for 37 of the 39 genes (Additional data files 1 and 2).

\section{Polymorphism detection}

In addition to gene amplification events, drug resistance may also arise through the emergence of SNPs; thus, we next tested whether the microarray could globally detect these polymorphisms (Figure 2). As with CNV detection, probes that mapped to only one location in the $3 \mathrm{D} 7$ reference genome were used for the analysis. A z-test was performed on the difference in log intensities of the reference and test isolate hybridization with a sliding window of three overlapping probes. If there was no difference in the genomic sequence hybridizing to the probes, then the intensity differences formed a normal distribution centered on a mean of zero. The standard deviation of the normal distribution was empirically determined by replicate hybridizations of rank-invariant probes for the z-test. The appearance of SNPs between the experimental genomic DNA and the reference isolate resulted in intensities that were higher in the reference isolate. Based on our empirical data, we classified probes with a $P$ value of less than $1 \times 10^{-8}$ and a higher mean in the reference hybridizations as ones containing polymorphisms in the isolate. Combining the data from the sliding windows of three probes enabled us to establish the boundaries within which the polymorphisms were contained. To predict the precise position of SNPs, we used an empirically determined model of loss of hybridization based on the SNP position in probes (Additional data file 3 [Figure S1]). An F-test was then performed with the model, based on the null hypothesis of the mean equaling zero, in order to position the polymorphism prediction at the peak of the $P$ value.

Our SNP prediction validation was performed by comparing the $3 \mathrm{D} 7$ reference isolate to $\mathrm{Dd} 2$ and $\mathrm{HB}_{3}$, which have recently been sequenced to greater than $8 \times$ coverage by the Broad Institute [12]. In our analysis, we included high quality SNPs (Phred score $>25$ for each SNP and more than 25 base pairs from either end of an alignment or base pair call with Phred score <20) that were present in four or more reads (Dd2), and excluded SNPs in hypervariable subtelomeric regions defined in recent re-sequencing efforts [12]. Because the $\mathrm{HB}_{3}$ genome was assembled before SNP detection in the work by Volkman and coworkers [12], we were unable to examine individual $\mathrm{HB}_{3}$ sequence reads to determine coverage and quality values for SNPs and thus had a larger set of SNPs about which we were less confident. Initial analyses with the test set limited to SNPs that mapped to at least three unique probes on the microarray provided coverage of $75.9 \%$ for coding regions and $41.2 \%$ for noncoding regions, but resulted in suboptimal detection rates (Additional data file 3 [Table S1]). Therefore, the test set was limited to SNPs that mapped to six or more unique probes on the array, which restricted our microarray coverage to $58.5 \%$ of coding regions and $23.2 \%$ of noncoding regions. This produced a validation set of 1,737 SNPs in Dd2 and 3,344 SNPs in HB3 (Additional data file 3 [Table S2] and Additional data file 4).

From this set of high-quality SNPs we were able to identify 91.1\% of SNPs (1,582 true positives, 155 false negatives) with a false discovery rate of $10.5 \%$ ( 185 false positives) when comparing two hybridizations of Dd2 with two reference hybridizations and using a $P$ value cutoff of $1 \times 10^{-8}$. Detection rate 


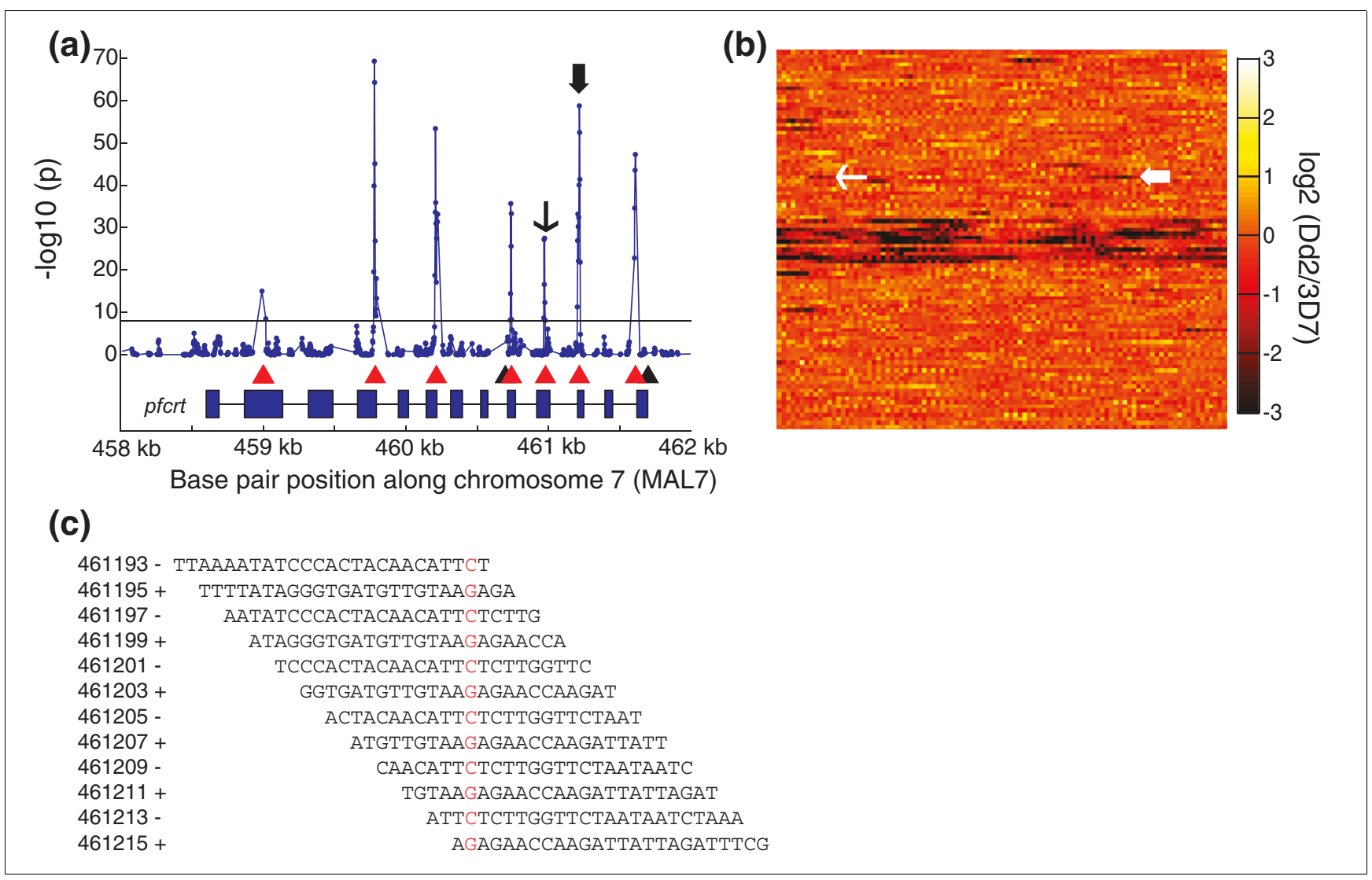

Figure 2

SNPs in Dd2. (a) Plot of - $\log _{10} P$ values for z-test (blue line) performed with Dd2 versus the 3D7 reference; detected are all of the reported single nucleotide polymorphisms (SNPs) in the pfcrt gene that were represented in the microarray (red triangles). Two SNPs were not detected (black triangles) because of the lack of unique probes to these positions. The thin and thick black arrows correspond to SNPs indicated in panel b. The $P$-value cutoff was $1 \times 10^{-8}$ (black line). (b) Visualization of probe intensity log ratios in Dd2 versus 3D7. Each probe is shown as a single pixel and is colored based on its log ratio. Continuous strips of dimmer pixels correspond to SNPs that map to multiple unique probes, as indicated by the thin and thick white arrows. (c) Probes were tiled through the genome with an offset of two to three base pairs of alternating strands. The position marked in red is the position of the SNP marked by the thick arrows in panels $a$ and $b$. The base pair positions on the left indicate the start of the probe with respect to the plus strand of chromosome 5 and its orientation.

was defined as the percentage of the SNP validation set that was confirmed by microarray analysis; false discovery rate was the percentage of SNPs detected by microarray that were not polymorphic by Broad Institute sequencing [12]. For the HB3 SNPs, we detected $85.0 \%$ of SNPs $(2,842$ true positives and 502 false negatives) with a false discovery rate of $16.7 \%$ (569 false positives) using the same $P$ value cutoff. The lower detection rate in $\mathrm{HB}_{3}$ may be due to the lack of sequence read information and thus less confidence in SNP identification from the original sequencing effort [12]. For Dd2 we also obtained similar lower detection rates when including lowerquality SNPs that had fewer than four reads. In Saccharomyces cerevisiae, Gresham and coworkers identified $96.2 \%$ of the published SNPs (944 out of 981) with a 95.9\% false discovery rate (22,082 false positives) [25]. With a more stringent prediction signal cutoff, Gresham and coworkers [25] were able to obtain an impressively low false discovery rate of $0.87 \%$ with $81.7 \%$ detection using the well characterized model organism $S$. cerevisiae. In comparison, with a $P$ value cutoff of $1 \times 10^{-5}$, we detected $93.9 \%$ of SNPs in Dd2 with a $57.6 \%$ false discovery rate. Although we were unable to achieve false discovery rates of less than $1 \%$ because of the incomplete sequencing of the isolates and the nonclonal nature of parasite cultures, our SNP identification and false discovery rates approach those of conventional sequencing the current gold standard (Tables 1 to 2 and Additional data file 3 [Figure S2]).

To scrutinize our false discovery and false negative rates, we used PCR amplification and subsequent sequencing of selected predicted polymorphisms with low $P$ values classified as 'false positives', as well as 'false negative' polymorphisms with high probe coverage that were missed by our method. Any 'false positives' arising from indels present in sequencing reports were manually filtered and excluded from false discovery rates for Dd2 $P$-value cutoffs of $1 \times 10^{-8}$ or $1 \times$ $10^{-10}$. Our results showed that $50 \%$ of the remaining highquality 'false positives' were actually polymorphisms that 
Table I

\begin{tabular}{|c|c|c|c|c|}
\hline$z$-test $P$ value & True positives & False positives & Detection rate (\%) & False discovery rate (\%) \\
\hline $1 \times 10^{-5}$ & 1,635 & 2,193 & 93.9 & 57.3 \\
\hline $1 \times 10^{-8}$ & I,582 & 185 & 91.1 & 10.5 \\
\hline $1 \times 10^{-10}$ & 1,532 & 39 & 88.0 & 2.48 \\
\hline
\end{tabular}

SNP, single nucleotide polymorphism.

were either different in the laboratory isolates that we analyzed or not detected by the re-sequencing project, similar to the $64 \%$ error rate described by Jiang and coworkers [26]. This suggests that our actual false discovery rate is approximately $6 \%$. We did not detect $8.9 \%$ of the reported SNPs with good probe coverage in Dd2, and for all of these 'false negatives' sequenced there indeed was a polymorphism that was not detected by our arrays. More stringent $P$-value cutoffs increased the false negative rate but improved the false discovery rate. Additionally, the prediction of SNP location also increased with more stringent $P$ values and higher density probe coverage. When the prediction signal contained more than ten probes that included the SNP, we were able to localize the position of the polymorphism prediction to within seven base pairs in more than $95 \%$ of cases and to within ten base pairs in more than $98 \%$ of cases. Even with weaker signals (four probes), we were able to localize approximately $95 \%$ of SNPs to within ten base pairs (Table 3 ).

\section{Comparison with previous studies}

Previous microarray-based studies of $P$. falciparum have assessed genetic variability in the parasite [22,26,32] but these microarrays were not designed with the probe density and coverage to detect small changes in the genome that result from in vitro evolution or drug resistance. For example, the recent study by Jiang and coworkers [26] reports rates of polymorphic probes for genes across various laboratory isolates but fails to detect the hallmark mutation resulting in the K76T mutation in pfcrt, presumably because of insufficient probe coverage. The authors did not report individual polymorphisms identified in each of the laboratory isolates, and thus it was not possible to compare our methods directly. Another advantage of our current study is that the overlapping nature of probes allowed us to use one or two microarray replicate hybridizations to detect polymorphisms instead of three to four hybridizations required in previous studies $[22,26]$.

\section{Detection of mutations in an engineered parasite line} Classical genetic approaches are essential for elucidating gene function, but the transfection process and the subsequent drug selection are population bottlenecks. Emerging parasites may contain changes elsewhere in the genome that are undetected by standard controls but contribute to phenotype. To determine systematically additional mutations that arose or were selected in an engineered parasite line, we hybridized genomic DNA from $3 \mathrm{D} 7^{\mathrm{attB}}$ to the microarray. The $3 \mathrm{D} 7^{\mathrm{attB}}$ line was generated from $3 \mathrm{D} 7$ by the insertion of an attB recombination site for Bxb1 integrase flanked by the human $d h f r$ gene as a selectable marker [33]. We detected a deletion of a subtelomeric region of chromosome 6 (1.38 to 1.41 megabases) and a polymorphism in the histone 2B gene (PF11_o062; Figure 3a and Additional data file 4). Sequencing revealed two novel point mutations resulting in a nonsynonymous mutation of A10oF in this highly conserved gene (Figure 3b), strengthening the importance of whole genomic screening for undetected mutations in engineered lines.

\section{Selection and characterization of fosmidomycin resistance}

Malarial parasites, unlike mammals, employ a nonmevalonate based 2-C-methyl-D-erythritol-4-phosphate (MEP) pathway for synthesis of essential isoprenoids such as ubiquinones and dolichols [34]. 1-Deoxy-D-xylulose 5-phosphate reductoisomerase (DXR), a key enzyme of the MEP pathway, catalyzes the conversion of 1-deoxy-D-xylulose 5-phosphate (DOXP) to MEP and is of particular therapeutic interest because of its requirement for isoprenoid synthesis in $P$. fal-

Table 2

Previously published SNP prediction results: yeast SNP call rates

\begin{tabular}{lllll}
\hline Prediction signal & True positives & False positives & Detection rate (\%) & False discovery rate (\%) \\
\hline$>0$ & 944 & 22,082 & 96.2 & 95.9 \\
$>5$ & 801 & 7 & 81.7 & 0.87 \\
$>5,347$ & 760 & 0 & 77.5 & 0.00 \\
\hline
\end{tabular}

Based on data from Gresham and coworkers [25]. SNP, single nucleotide polymorphism. 
Table 3

\begin{tabular}{lllll}
\hline \multicolumn{4}{l}{ Accuracy of Dd2 SNP positioning } \\
\hline Signal probes & $\mathbf{0}$ bp (\%) & $\mathbf{\pm} \mathbf{2}$ bp (\%) & $\mathbf{\pm} \mathbf{5}$ bp (\%) & $\mathbf{\pm} 10$ bp (\%) \\
\hline 4 to 5 & 8.9 & 50.0 & 83.7 & 94.7 \\
6 to 7 & 13.5 & 60.7 & 88.6 & 97.8 \\
8 to 10 & 17.7 & 67.4 & 92.1 & 98.2 \\
II to 13 & 18.2 & 69.3 & 91.1 & 98.1 \\
\hline
\end{tabular}

bp, base pairs; SNP, single nucleotide polymorphism.

ciparum as well as several pathogenic eubacteria including Bacillus anthracis, Helicobacter pylori, Yersinia pestis, and Mycobacterium tuberculosis [35]. Recent clinical studies with the specific DXR inhibitor fosmidomycin have shown that this agent is well tolerated in humans and can cure P. falciparum infection, either alone or more potently in combination with clindamycin or artesunate [36-38]. These trials have yet to report resistance to fosmidomycin, including in areas with a high prevalence of parasite resistance to several other clinically used antimalarials.

To gain insight into how fosmidomycin resistance could evolve in $P$. falciparum, Dd2 parasites were exposed to drug in vitro and selected under stepwise increases in concentration. Two fosmidomycin-resistant clones, named FOS-RDd2CL1 and FOS-RDd2-CL2, were isolated from two separate flasks. These were then subjected to 72-hour [3H]hypoxanthine incorporation assays to determine the degree of resistance. Fosmidomycin 50\% inhibitory concentration $\left(\mathrm{IC}_{50}\right)$ values for FOS-RDd2-CL1 and FOS-RDd2-CL2 were 2,219 \pm 136 and 2,623 \pm $78 \mathrm{nmol} / \mathrm{l}$, respectively, compared with an approximately eightfold lower $\mathrm{IC}_{50}$ value of $307 \pm 49 \mathrm{nmol} / \mathrm{l}$ for the parental Dd2 line (Figure 4a). Fosmidomycin-resistant lines exhibited no change in their $\mathrm{IC}_{50}$ value for chloroquine compared with Dd2 (data not shown).
To confirm that fosmidomycin resistance was related to the MEP pathway, infected erythrocytes were pretreated for 24 hours with fosmidomycin and the parasites released by saponin treatment. Intact erythrocyte-free parasites were then metabolically labeled with [2-14C]pyruvic acid in the presence of fosmidomycin. As expected, the Dd2 parental line showed DOXP accumulation while MEP synthesis was reduced in a dose-dependent way in treated parasites (Figure 4b). In contrast, the synthesis of DOXP and MEP was unaffected by drug in fosmidomycin-resistant parasites (Figure 4b). The fact that DOXP did not accumulate in a dosedependent manner in Dd2 could be due to negative feedback regulation, as previously observed in P. falciparum [39,40]. As a control, the synthesis of adenosine metabolites through the purine pathway was analyzed under the same treatment conditions using [2-3H]adenosine as the metabolic precursor (Additional data file 3 [Figure $\mathrm{S}_{3} \mathrm{AB}$ ]). No significant differences in [2-3H]adenosine uptake were observed between untreated and fosmidomycin-treated parasites both in Dd2 and FOS-RDd2-CL1. Similarly, no significant differences in parasite growth were observed during the 24-hour treatment (Additional data file 3 [Figure $\mathrm{S}_{3} \mathrm{C}$ ]). To test the stability of the fosmidomycin-resistant phenotype, frozen stocks of the FOS-R $\mathrm{R}^{\mathrm{Dd} 2-\mathrm{CL} 1}$ clone were thawed and parasites maintained in the absence of drug pressure for 6 weeks. Fosmidomycin assays with this parasite line yielded an $\mathrm{IC}_{50}$ value of $2,958 \pm$ $101 \mathrm{nmol} / \mathrm{l}$, which was comparable with in vitro selected resistant lines maintained under drug (Figure 4a). These studies demonstrate that fosmidomycin resistance can be readily and stably acquired in vitro by selective pressure on culture-adapted malarial parasites.

To investigate the genetic basis of this drug resistance, DNA from these fosmidomycin-resistant mutants and the parental Dd2 line were hybridized to the arrays. Unexpectedly, we identified a single large amplification event in the genome, on chromosome 14 of the fosmidomycin-resistant clones com-

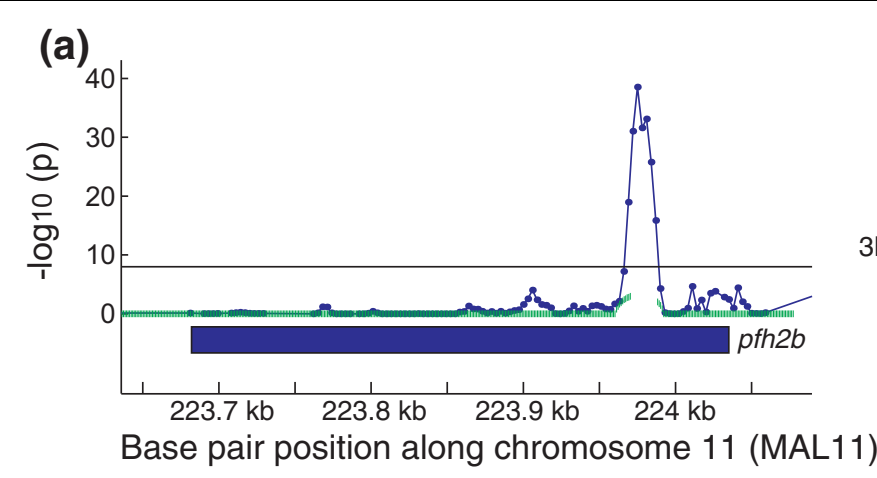

(b)

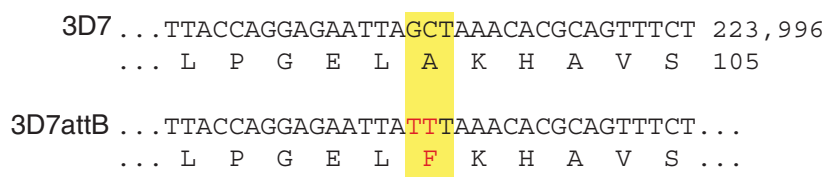

Figure 3

Mutation in 3D7attB histone 2B. (a) Plot of -log $P$-value for z-test (blue line) performed with 3D7attB versus the 3D7 reference detected a polymorphism in the histone $2 \mathrm{~B}$ gene with a $P$-value cutoff of $\mathrm{I} \times 10^{-8}$ (black line). (b) Sequencing of the histone $2 \mathrm{~B}$ gene in $3 \mathrm{D} 7$ attB revealed two consecutive point mutations resulting in a coding nonsynonymous mutation. 


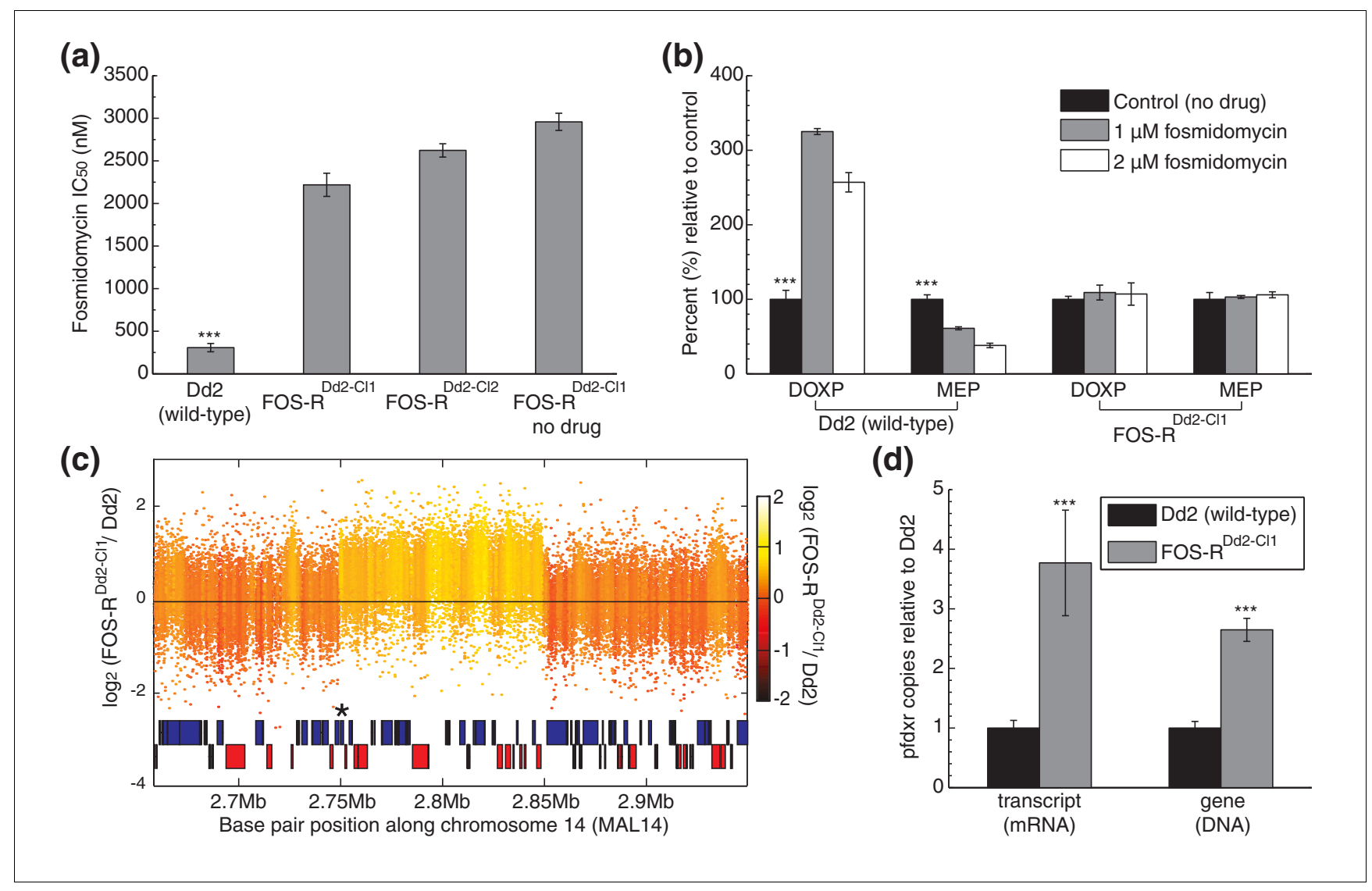

\section{Figure 4}

Fosmidomycin resistance. (a) Fosmidomycin 50\% inhibitory concentration $\left(\mathrm{IC}_{50}\right)$ values (means and standard deviations) were calculated from independent $\left[{ }^{3} \mathrm{H}\right]$ hypoxanthine assays in duplicate; the number of assays $(n)$ is indicated in parenthesis. Drug susceptibility profiles of Dd2 (wild-type) $(n=$ 7), FOS-RDd2-CLI $(n=5)$, FOS-RDd2-CL2 $(n=3)$, and control FOS-RDd2-CLI-no drug $(n=3)$. Tests for significant differences between the parental and drugresistant isolates were performed using analysis of variance with Bonferroni posttests with $\alpha=0.00 \mathrm{I}$; significant differences $(P$ value $<0.00 \mathrm{I})$ are marked with three asterisks ${ }^{(* * *)}$. (b) Effect of fosmidomycin on I-deoxy-D-xylulose 5-phosphate (DOXP) and 2-C-methyl-D-erythritol-4-phosphate (MEP) biosynthesis in intact erythrocyte-free parasites exposed to fosmidoymicin for 24 hours and then labeled with $\left[2-{ }^{14} \mathrm{C}\right]$ pyruvic acid as a metabolic precursor. Means and standard deviations were calculated from four independent experiments performed in duplicate. Differences between control (no drug) Dd2 and fosmidomycin-treated Dd2 were significant by Student's $t$-test for DOXP $\left(I \mu \mathrm{mol} / \mathrm{l}\right.$ drug: $P$ value $I \times 10^{-8} ; 2 \mu \mathrm{mol} / \mathrm{I}$ drug: $P$ value $\left.I \times 10^{-6}\right)$ and MEP $\left(I \mu \mathrm{mol} / / \mathrm{l}\right.$ drug: $P$ value $4 \times 10^{-6} ; 2 \mu \mathrm{mol} / /$ drug: $P$ value $\left.5 \times 10^{-8}\right)$ and are marked with three asterisks $(* * *)$. (c) To generate the plot, the log 2 ratio of the intensity of each unique probe in FOS-RDd2-CLI was divided by the intensity of each unique probe in Dd2. The probe log ratios were colored by the moving average over a 500 base pair window as indicated in the color bar. The amplification was approximately $100 \mathrm{~kb}$ and contained 23 genes including pfdxr (marked with an asterisk). (d) Quantitative RT-PCR analysis of $p f d x r$ transcript level and quantitative PCR analysis gene copy number in FOS-RDd2-CLI compared with Dd2. The means and standard deviations of two independent quantitative RT-PCR and quantitative PCR assays are represented. Significance differences $(P$ value $<0.00 \mathrm{I})$ in $\Delta \mathrm{Ct}$ values were determined by Wilcoxon rank sum test and are marked with three asterisks $(* * * *)$.

pared with Dd2. This CNV contained 23 genes (PF14_o641 to PF14_0663) and was approximately $100 \mathrm{~kb}$ in size (Figure $4 \mathrm{~b}$ and Additional data file 2). The first gene in the amplification event was identified as $p f d x r$, the putative target of fosmidomycin in Plasmodium, and the amplification event included the entire upstream intergenic region for this gene. The $\log _{2}$ ratio of FOS-R ${ }^{\mathrm{Dd} 2-\mathrm{CL} 1} / \mathrm{Dd} 2$ intensity for the region was approximately 0.7, suggesting the presence of three copies of this region. Quantitative RT-PCR demonstrated an approximately 3.8-fold increase in the $p f d x r$ transcript level and a approximately 2.7-fold increase in gene copy number in FOS$\mathrm{R}^{\mathrm{Dd} 2-\mathrm{CL} 1}$ when compared with Dd2 (Figure 4d). These investigations suggest that fosmidomycin pressure selected for par- asites that had amplified the $p f d x r$ gene. Finally, the entire open reading frames of $p f d x r$ and $p f d x s$ (DOXP synthase, MAL13P1.186), the most likely candidate resistant determinants in the mevalonate-independent isoprenoid synthesis pathway, were sequenced and analyzed for the presence of mutations. No sequence changes were observed in comparison with the wild-type parasite line.

\section{Development of software tools}

We created a powerful MATLAB-based graphical user interface that implements our methods to visualize SNPs, perform SNP prediction, and visualize gene CNVs, allowing researchers to interpret the microarray data. This software is freely 
available as a standalone application without restrictions for Mac OS X and Windows platforms from our website [41].

\section{Discussion}

Recent studies suggest that CNVs of genes, involved in diverse processes from cell cycle regulation to sexual differentiation, may be a common strategy used by $P$. falciparum to overcome environmental stresses and drug pressure $[22,31,42]$. The amplification of GTP cyclohydrolase I, the first enzyme of the folate biosynthesis pathway, is hypothesized to shuttle more substrate into this pathway in the presence of various antifolate antimalarials and was missed in the sequencing efforts [22]. This is not surprising because sequencing the human genome missed a large number of CNVs subsequently described by various microarray analyses [43-46]. Compared with the microarrays used in all of these studies, our method not only allows for more powerful statistics with hundreds to thousands of unique probes for most genes, but it also allows for accurate break point mapping with probes to intergenic regions. We applied our method to fosmidomycin-resistant parasites and identified an increase in gene copy number of the putative target $p f d x r$ as a plausible mechanism for in vitro derived resistance. This approach can be extended to additional antimalarial compounds to determine candidate mechanisms of resistance and potentially identify drug targets when the mechanism of action is unknown.

Although CNVs in pfmdr1 and pfgch1 have been reported, the amplification of $p f d x r$ is the first example of resistance due to the amplification of the target enzyme of a drug in Plasmodium. These amplification events may be common methods for parasites to increase transcription of important genes in light of recent studies confirming the inflexibility of the programmed asexual stage transcriptome to respond to drug pressure [47] because parasites have evolved in a relatively stable environment of the human host. Elucidating the mechanism of resistance to fosmidomycin is important in view of its promising role in clinical combination therapies [36-38]. Although the volume of blood obtained with a finger stick may not yield enough parasite DNA to do these experiments, 5 to $10 \mathrm{ml}$ of leukocyte-depleted infected blood may yield enough material for a successful hybridization. In additional, clonal field isolates may be pushed into short-term culture for characterization by microarray.

Our method allows for rapid and accurate detection of SNPs in the parasite, as illustrated by our detection of known SNPs in the established drug resistance genes dhfr-ts and pfcrt in Dd2 (Figure 2a, b). This method achieved greater than 90\% SNP detection with low false positive rates of about $10 \%$, similar to those reported by re-sequencing efforts [12]. Polymorphisms identified by microarray improve the confidence of SNPs identified by sequencing, and perhaps these two methods can be implemented in a complementary way for large- scale detection of genetic variation. The existence of up to 13 probes covering a single base pair location provided the equivalent of multiple-fold coverage that sequencing can provide. Insertions and deletions (indels) are common mutations that we also wished to evaluate by microarray. Unfortunately, indels were not systematically reported by re-sequencing efforts, and thus we were unable to determine the accuracy of indel identification by microarrays compared with sequencing. However, we did find that many 'false positives' were actually insertions or deletions present in the sequencing reports, suggesting that our method can also accurately detect indels. We also identified nonsynonymous point mutations in the engineered parasite line $3 \mathrm{D} 7^{\text {attB }}$, illustrating the utility of our microarray for discovering undetected and unexpected genetic changes that may arise or be selected during genetic manipulation and subsequent selection of parasites.

Traditional sequencing methods, like those used to sequence all $P$. falciparum isolates to date, are considered to be the gold standard. Yet their consensus accuracies of $99.99 \%$ would still result in thousands of potential errors. Furthermore, shotgun sequencing methods involve transforming Escherichia coli with parasite DNA, which can be difficult with $P$. falciparum because of its extremely high AT content. PCR amplification steps may also bias the results, and traditional sequencing methods have missed CNVs that are an important mechanism for drug resistance in $P$. falciparum and other species, including humans [43-45]. Next generation sequencing technologies are quickly becoming more affordable and are beginning to be used to assess genetic variability in a number of organisms with SNP detection rates that are comparable [48-50] to those of our microarray-based method. However, these methods also have their caveats. With a highly repetitive AT-rich genome like $P$. falciparum, masking or filtering steps may limit the detection of polymorphisms in many intergenic regions and coding regions. Our hybridization-based method achieves detection rates similar to those of sequencing and is much faster, requiring a single overnight experiment followed by rapid computational analysis with results presented in a form readily accessible to researchers.

\section{Conclusions}

Our whole-genome approach will prove particularly helpful when elucidating mechanisms of drug resistance in vitro or in clinical isolates of $P$. falciparum, as demonstrated by the identification of $p f d x r$ amplification in fosmidomycin resistance. This method can also be applied to screening populations of $P$. falciparum and tracking the spread of drug resistance, to discover the genetic basis of other phenotypic changes, and to address more fundamental questions in $P$. falciparum biology, such as the basal rates of point mutations and CNVs in laboratory culture. By probing genetic variability in $P$. falciparum with a single hybridization in a single overnight experiment, this tiled microarray methodology will 
allow researchers using the accompanying software to quickly and accurately ascribe phenotypic variations to changes at the gene level. This will facilitate a deeper understanding of how the parasite is evolving worldwide both in field and laboratory-adapted field isolates, supporting the new drive to reduce the burden of malaria more effectively.

\section{Materials and methods DNA methods}

Cultured isolates of 3D7 (MRA-151), Dd2 (MRA-156), HB3 (MRA-155), and 3D7 attB (MRA-845) were obtained from the Malaria Research and Reference Reagent Resource Center (MR4; American Type Culture Collection, Manassas, VA, USA). P. falciparum parasite lines were propagated in human erythrocytes as previously described [33,51]; Dd2 was freshly cloned from the MR4 isolate. Genomic DNA was isolated by standard phenol-chloroform extraction. Fifteen micrograms of genomic DNA from each isolate and $10 \mathrm{ng}$ each of Bio B, Bio C, Bio D, and Cre Affymetrix control plasmids (Affymetrix Inc., Santa Clara, CA, USA) were fragmented with DNaseI and end-labeled with biotin [52]. The samples were hybridized to the microarrays overnight at $45^{\circ} \mathrm{C}$ in Affymetrix buffers, washed, and scanned [22].

Background subtraction and microarray normalization Background subtraction was performed for each array using Affymetrix eukaryotic background control probes. Because of the varying background hybridization of probes based on their GC content, the background probes were split into bins based on GC content, and the mean for each bin was calculated. The appropriate background value, based on GC content, was subtracted from probes used for the analyses. As described above, the arrays were normalized to a synthetic baseline array constructed by taking the means across all probes for all arrays used in the experiment. The probe intensities were log transformed and placed into 100 equally sized and spaced bins. After removing outliers, the median for each bin was set to the median of the corresponding bin in the synthetic baseline microarray.

\section{Probe selection}

The microarray contains probes to the $P$. falciparum $3 \mathrm{D} 7$ nuclear, mitochondrial, and apicoplast genomes, as well as isolate-specific probes for $\mathrm{HB}_{3}$ and $\mathrm{Dd} 2, P$. knowlesi specific probes, and eukaryotic control probes (standard Affymetrix and Arabidopsis thaliana). Unique probe mapping was determined by blasting $P$. falciparum probe sequences against the reference genome (PlasmoDB version 5.3) and determining the number of perfect matches. We excluded from our analysis any probes that had more than one perfect match.

\section{Nucleotide sequencing}

Primers were designed to amplify 400 to 500 base pairs of Dd2 genomic DNA with the false positive or false negative position near the center. Independent PCR products were sequenced by ABI sequencing (Applied Biosystems Inc., Foster City, CA, USA).

\section{In vitro selection of fosmidomycin-resistant parasites and antimalarial drug assays}

The $P$. falciparum Dd2 line (a mefloquine-resistant derivative of W2 originally from Indochina) was used for the selection of fosmidomycin resistance. For the drug selection experiment, approximately $2 \times 10^{10}$ mixed stage parasites were exposed to $100 \mathrm{nmol} / \mathrm{l}$ of fosmidomycin for 5 days. Cultures were maintained carefully by smearing every day and feeding twice daily with drug-containing RPMI media. After initial drug treatment, fosmidomycin drug concentration was increased to $400 \mathrm{nmol} / \mathrm{l}$ and kept at this level for the following 7 days. At this drug concentration, dying asexual-stage parasites were observed by microscopic examination of Giemsa-stained smears. The fosmidomycin drug selection level was then increased to $700 \mathrm{nmol} / \mathrm{l}$, which eliminated asexual stage parasites from detection by microscopy, and cultures were further maintained at this drug level throughout the experiment. Reappearance of healthy asexual stage parasites growing in the presence of drug were observed approximately 6 weeks after the start of the selection experiment. Once parasitemia reached $2 \%$ to $3 \%$, frozen stocks of fosmidomycin-resistant parasites were prepared using Glycerolyte 57 (Baxter Healthcare, Deerfield, IL, USA). During the entire selection process $30 \%$ to $40 \%$ red blood cells were replaced with freshly washed cells once a week. Drug selected parasites were cloned by limiting dilution in 96-well tissue culture plates in the presence of $700 \mathrm{nmol} / 1$ of fosmidomycin, with an inoculum of 0.5 infected red blood cells per well. Parasite clones were detected after 3 weeks of growth using the $P$. falciparum lactate dehydrogenase-specific Malstat assay [53].

Parasites were phenotypically characterized for their drug susceptibility profiles using $[3 \mathrm{H}]$ hypoxanthine incorporation assays, as described previously [54]. The response to fosmidomycin and chloroquine was measured in vitro in 96-well plates using 72-hour [3H]hypoxanthine assays, starting with an initial parasitemia of $0.4 \%$ to $0.5 \%$. $\mathrm{IC}_{50}$ values were calculated using linear regression $[54,55]$.

\section{Metabolite profiling}

One cycle after sorbitol synchronization, predominantly ringstage cultures at about 10\% parasitemia were treated for 24 hours with $1 \mu \mathrm{mol} / \mathrm{l}$ or $2 \mu \mathrm{mol} / \mathrm{l}$ fosmidomycin. Untreated cultures were incubated in parallel. After treatment, parasites were isolated from their host erythrocytes by incubating with 20 pellet volumes of $0.015 \%$ saponin in phosphate-buffered saline (PBS), and then washed three times with PBS at room temperature. Parasitemia and parasite morphology were determined by microscopic analysis of Giemsa-stained blood smears immediately before and after saponin treatment. Untreated or treated intact erythrocyte-free parasites were 
resuspended in RPMI 1640 medium and labeled for 1 hour with $14 \mu \mathrm{mol} / 1$ [2-14C]pyruvic acid (10 to $40 \mathrm{mCi} / \mathrm{mmol}$; Perkin Elmer, Waltham, MA, USA) or $1 \mu \mathrm{mol} / 1$ [2-3H]adenosine (23 Ci/mmol; Amersham Biosciences, Piscataway, NJ, USA) in the absence (controls) or presence of fosmidomycin. After incubation, parasites were centrifuged and washed twice with ice-cold PBS. Parasites were immediately extracted with ethanol/water (1:1 vol/vol; $1 \times 0.3 \mathrm{ml}$ at $55^{\circ} \mathrm{C}$ for 1.5 hours) [39] for subsequent high-performance liquid chromatography (HPLC) analysis of DOXP and MEP metabolites. Purine metabolites were extracted by perchloric acid treatment. Briefly, samples were mixed 1:6 (vol/vol) with $0.5 \mathrm{~mol} / \mathrm{l}$ $\mathrm{HClO}_{4}$, vigorously mixed, and incubated for 20 minutes at $4^{\circ} \mathrm{C}$. Samples were centrifuged and supernatants were neutralized with $5 \mathrm{~mol} / \mathrm{l} \mathrm{KOH}$ for 20 minutes at $4{ }^{\circ} \mathrm{C}$. All extracts were filtered through YM-10 Centricon columns (MW retention = 10000; Amicon, Millipore, Billerica, MA, USA). Analyses of metabolites were accomplished by using $2 \times 10^{8}$ parasites.

At the beginning of treatment, $1 \mathrm{ml}$ culture from each condition was used in [3H]hypoxanthine incorporation assays to test growth inhibition. After 12 hours of treatment, $[3 \mathrm{H}]$ hypoxanthine was added at a final concentration of 5 $\mu \mathrm{Ci} / \mathrm{ml}$. After an additional 12 hours of incubation, cells were harvested according to the method described by Desjardins and colleagues [54].

DOXP and MEP intermediates were analyzed by HPLC as described previously [39]. Briefly, the ethanol/water fractions were analyzed using a reverse-phase column (Luna $\mathrm{C}_{18}$ [2], $150 \times 4.6 \mathrm{~mm}, 3 \mu \mathrm{m}$; Phenomenex, Torrance, CA, USA). The eluants were $20 \mathrm{mmol} / \mathrm{l} N, N$-dimethylhexylamine in $10 \%$ methanol with the $\mathrm{pH}$ adjusted to 7.0 with formic acid (solution A) and 50\% methanol containing $2 \mathrm{mmol} / \mathrm{l} \mathrm{N}, \mathrm{N}$ dimethylhexylamine, $\mathrm{pH}$ 7.0 (solution B). The HPLC gradient was $10 \%$ to $50 \%$ methanol in 50 minutes. The eluant was monitored at $270 \mathrm{~nm}$ at a flow rate of $0.75 \mathrm{ml} /$ minute. The adenosine metabolites inosine, hypoxanthine and inosine monophosphate were analyzed in a reverse-phase (Luna $\mathrm{C}_{18}$ [2], $150 \times 4.6 \mathrm{~mm}, 3 \mu \mathrm{m}$; Phenomenex, Torrance, CA, USA) ion-pair HPLC system. The mobile phases were 8 $\mathrm{mmol} / \mathrm{l}$ tetrabutylammonium bisulfate (Fluka, Sigma Aldrich, St. Louis, MO, USA) and $100 \mathrm{mmol} / \mathrm{KH}_{2} \mathrm{PO}_{4}$ with the $\mathrm{pH}$ adjusted to 6.0 with $\mathrm{KOH}$ (solution $\mathrm{A}$ ), and $30 \%$ acetonitrile containing $8 \mathrm{mmol} / \mathrm{l}$ tetrabutylammonium bisulfate and $100 \mathrm{mmol} / 1 \mathrm{KH}_{2} \mathrm{PO}_{4}(\mathrm{pH} 6)$ as solution $\mathrm{B}$. The HPLC gradient was from $0 \%$ to $100 \%$ solution $\mathrm{B}$ in 20 minutes. The eluant was monitored at $254 \mathrm{~nm}$ and the flow rate was $1 \mathrm{ml} /$ minute. Aliquots from both HPLC systems were collected based on UV detection of internal standards and subjected to liquid scintillation counting. For the comparison of the influence of fosmidomycin treatment on the biosynthesis of the different metabolites, the same numbers of treated or untreated parasites were analyzed.

\section{Quantification of $p f d x r$ copy number and transcript levels}

Real-time PCR and real-time RT-PCR methods were employed to quantify $p f d x r$ gene copy number and transcript levels respectively in fosmidomycin-resistant and parental lines. Genomic DNA extractions were performed using Qiagen DNeasy kits (Qiagen, Hilden, Germany), and RNA samples were prepared from the trophozoite-stage synchronized parasites using Trizol (Invitrogen, Carlsbad, CA, USA). DNase-treated RNA was reverse transcribed using the SuperScript ${ }^{\circledR}$ III First-Strand Synthesis System (Invitrogen, Carlsbad, CA, USA). Genomic DNA and cDNA templates (at concentrations $1,0.5,0.25,0.125,0.625,0.0312$, and 0.01562 ng) were PCR amplified using the QuantiTect SYBR Green PCR Kit (Qiagen, Hilden, Germany) with $p f d x r$-specific primers (forward: 5'-TCAAGAACTTGCGATATTATAGAGG; reverse: 5'-TTGGCTCAGGTTTCAACTCTTACAT) or actinspecific primers (forward: 5'-AGCAGCAGGAATCCACACA; reverse: $\quad 5^{\prime}$-TGATGGTGCAAGGGTTGTAA). The $2^{-\Delta \Delta \mathrm{Ct}}$ method was employed to assess the copy number and transcript level (Applied Biosystems Inc., Foster City, CA, USA).

\section{Database version}

All gene information and base pair positions were taken from PlasmoDB version 5.3 [56].

\section{Microarrays and probe definition files}

The microarray probe definition file, raw microarray data (CEL files), and result files from our analysis are available for download from our website [41]. Microarrays can only be custom ordered in bulk quantities, and thus upon request, the authors will coordinate groups wishing to obtain microarrays and have authorized Affymetrix to sell the microarrays to anyone who wishes to buy them.

\section{Abbreviations}

CNV: copy number variation; DOXP: 1-deoxy-D-xylulose 5phosphate; DXR: 1-deoxy-D-xylulose 5-phosphate reductoisomerase; HPLC: high-performance liquid chromatography; $\mathrm{IC}_{50}: 50 \%$ inhibitory concentration; kb: kilobase; MEP: 2-Cmethyl-D-erythritol-4-phosphate; MOID: match-only integral distribution; MR4: Malaria Research and Reference Reagent Resource Center; PBS: phosphate-buffered saline; RTPCR: reverse transcription polymerase chain reaction; SNP: single nucleotide polymorphism.

\section{Authors' contributions}

NVD, ABSS, MBC, DAF, and EAW conceived and designed the experiments. NVD, ABSS, MBC, SJW, SERB, RTE, and DP performed the experiments. SB and EAW designed the microarray. NVD developed analysis methods and analyzed the microarray data. SJW, DJP, SKV, DFW, and YZ contributed materials, data, and ideas. NVD, ABSS, MBC, DAF, and 
EAW wrote the paper. SJW, SERB, RTE, DJP, and SKV revised drafts of the paper.

\section{Additional data files}

The following additional data are included with the online version of this article: a table listing MOID gene present calls and average $\log _{2}$ ratios for Dd2, HB3, $3 \mathrm{D}^{\text {attB }}$, and FOS-R ${ }^{\mathrm{Dd} 2-}$ CL1 (Additional file 1); a table listing CNVs predicted by our algorithm in Dd2, HB3, 3 ${ }^{\mathrm{D}} 7^{\text {attB }}$, and FOS-R ${ }^{\mathrm{Dd} 2-\mathrm{CL} 1}$ (Additional data file 2); supplementary tables $\mathrm{S} 1$ and $\mathrm{S} 2$, and figures $\mathrm{S} 1$ to $\mathrm{S}_{3}$ (Additional data file 3); and a table listing polymorphisms predicted by our algorithm in $\mathrm{Dd} 2, \mathrm{HB} 3,3 \mathrm{D} 7^{\text {attB }}$, and FOR$\mathrm{R}^{\mathrm{Dd} 2-\mathrm{CL} 1}$ (Additional data file 4).

\section{Acknowledgements}

We thank $V$ Bounkeua for helpful discussions and careful reading of the manuscript and C Kidgell for genomic DNA hybridization samples. This work was supported by grants to EAW from the US National Institutes of Health (AI059472) and the WM Keck Foundation, and a grant to DF from the US National Institutes of Health (Al060342; PI Jim Sacchettini). Drug discovery research at the Genomics Institute of the Novartis Research Foundation is supported by funds from the Wellcome Trust and Medicines for Malaria Venture.

\section{References}

I. Ekland EH, Fidock DA: Advances in understanding the genetic basis of antimalarial drug resistance. Curr Opin Microbiol 2007, 10:363-370.

2. Ringwald P: Susceptibility of Plasmodium falciparum to antimalarial drugs: Report on global monitoring: 1996-2004 Geneva, Switzerland: World Health Organization; 2005.

3. Richie TL: High road, low road? Choices and challenges on the pathway to a malaria vaccine. Parasitology 2006, I33:SI I3-SI 44.

4. Thera MA, Doumbo OK, Coulibaly D, Diallo DA, Kone AK, Guindo AB, Traore K, Dicko A, Sagara I, Sissoko MS, Baby M, Sissoko M, Diarra I, Niangaly A, Dolo A, Daou M, Diawara SI, Heppner DG, Stewart VA, Angov E, Bergmann-Leitner ES, Lanar DE, Dutta S, Soisson L, Diggs CL, Leach A, Owusu A, Dubois M-C, Cohen J, Nixon JN, et al: Safety and immunogenicity of an AMA-I malaria vaccine in Malian adults: results of a phase I randomized controlled trial. PLOS ONE 2008, 3:e I 465.

5. Weisman JL, Liou AP, Shelat AA, Cohen FE, Kiplin Guy R, DeRisi JL: Searching for new antimalarial therapeutics amongst known drugs. Chem Biol Drug Des 2006, 67:409-416.

6. Chong CR, Chen X, Shi L, Liu JO, Sullivan DJ: A clinical drug library screen identifies astemizole as an antimalarial agent. Nat Chem Biol 2006, 2:415-416.

7. Plouffe D, Brinker A, McNamara C, Henson K, Kato N, Kuhen K, Nagle A, Adrian F, Matzen JT, Anderson P, Nam T-g, Gray NS, Chatterjee A, Janes J, Yan SF, Trager R, Caldwell JS, Schultz PG, Zhou Y, Winzeler EA: In silico activity profiling reveals the mechanism of action of antimalarials discovered in a high-throughput screen. Proc Natl Acad Sci USA 2008, 105:9059-9064.

8. Wellems TE, Panton LJ, Gluzman IY, do Rosario VE, Gwadz RW, Walker-Jonah A, Krogstad DJ: Chloroquine resistance not linked to mdr-like genes in a Plasmodium falciparum cross. Nature 1990, 345:253-255.

9. Fidock DA, Nomura T, Talley AK, Cooper RA, Dzekunov SM, Ferdig MT, Ursos LMB, bir Singh Sidhu A, Naudé B, Deitsch KW, Su X-z, Wootton IC, Roepe PD, Wellems TE: Mutations in the $\boldsymbol{P}$. falciparum digestive vacuole transmembrane protein PfCRT and evidence for their role in chloroquine resistance. Mol Cell 2000, 6:86|-87|.

10. Sidhu ABS, Verdier-Pinard D, Fidock DA: Chloroquine resistance in Plasmodium falciparum malaria parasites conferred by $p f c r t$ mutations. Science 2002, 298:210-213.

II. Hunt P, Afonso A, Creasey A, Culleton R, Sidhu ABS, Logan J, Valder- ramos SG, McNae I, Cheesman S, Rosario Vd, Carter R, Fidock DA, Cravo $P$ : Gene encoding a deubiquitinating enzyme is mutated in artesunate- and chloroquine-resistant rodent malaria parasites\&sect. Mol Microbiol 2007, 65:27-40.

12. Volkman SK, Sabeti PC, DeCaprio D, Neafsey DE, Schaffner SF, Milner DA, Daily JP, Sarr O, Ndiaye D, Ndir O, Mboup S, Duraisingh MT, Lukens A, Derr A, Stange-Thomann N, Waggoner S, Onofrio R, Ziaugra L, Mauceli E, Gnerre S, Jaffe DB, Zainoun J, Wiegand RC, Birren BW, Hartl DL, Galagan JE, Lander ES, Wirth DF: A genome-wide map of diversity in Plasmodium falciparum. Nat Genet 2007, 39:113-119.

13. Jeffares DC, Pain A, Berry A, Cox AV, Stalker J, Ingle CE, Thomas A Quail MA, Siebenthall K, Uhlemann A-C, Kyes S, Krishna S, Newbold C, Dermitzakis ET, Berriman M: Genome variation and evolution of the malaria parasite Plasmodium falciparum. Nat Genet 2007, 39:120-125

14. Mu J, Awadalla P, Duan J, McGee KM, Keebler J, Seydel K, McVean GAT, Su X-z: Genome-wide variation and identification of vaccine targets in the Plasmodium falciparum genome. Nat Genet 2007, 39: 126-130.

15. Wootton JC, Feng X, Ferdig MT, Cooper RA, Mu J, Baruch DI, Magill AJ, Su X-z: Genetic diversity and chloroquine selective sweeps in Plasmodium falciparum. Nature 2002, 4I 8:320-323.

16. Price RN, Uhlemann A-C, Brockman A, McGready R, Ashley E, Phaipun L, Patel R, Laing K, Looareesuwan S, White NJ, Nosten F, Krishna S: Mefloquine resistance in Plasmodium falciparum and increased pfmdrl gene copy number. Lancet 2004, 364:438-447.

17. Sidhu ABS, Uhlemann A-C, Valderramos SG, Valderramos J-C, Krishna S, Fidock DA: Decreasing pfmdrl copy number in Plasmodium falciparum malaria heightens susceptibility to mefloquine, lumefantrine, halofantrine, quinine, and artemisinin. Infect Dis 2006, 194:528-535.

18. Jiang $H$, Joy $D$, Furuya $T$, Su $X z$ : Current understanding of the molecular basis of chloroquine-resistance in Plasmodium falciparum. J Postgrad Med 2006, 52:27I-276.

19. Reed MB, Saliba KJ, Caruana SR, Kirk K, Cowman AF: Pgh I modulates sensitivity and resistance to multiple antimalarials in Plasmodium falciparum. Nature 2000, 403:906-909.

20. Gregson A, Plowe CV: Mechanisms of resistance of malaria parasites to antifolates. Pharmacol Rev 2005, 57: I 17- 145.

21. Sidhu ABS, Sun Q, Nkrumah LJ, Dunne MW, Sacchettini JC, Fidock $\mathrm{DA}$ : In vitro efficacy, resistance selection, and structural modeling studies implicate the malarial parasite apicoplast as the target of azithromycin. J Biol Chem 2007, 282:2494-2504.

22. Kidgell C, Volkman SK, Daily J, Borevitz JO, Plouffe D, Zhou Y, Johnson JR, Le Roch KG, Sarr O, Ndir O, Mboup S, Batalov S, Wirth DF, Winzeler EA: A systematic map of genetic variation in Plasmodium falciparum. PLoS Pathog 2006, 2:e57.

23. Nair S, Miller B, Barends M, Jaidee A, Patel J, Mayxay M, Newton P, Nosten Fo, Ferdig MT, Anderson TJC: Adaptive copy number evolution in malaria parasites. PLoS Genetics 2008, 4: e 000243.

24. Gardner MJ, Hall N, Fung E, White O, Berriman M, Hyman RW, Carlton JM, Pain A, Nelson KE, Bowman S, Paulsen IT, James K, Eisen JA, Rutherford K, Salzberg SL, Craig A, Kyes S, Chan M-S, Nene V, Shallom SJ, Suh B, Peterson J, Angiuoli S, Pertea M, Allen J, Selengut J, Haft $D$, Mather MW, Vaidya AB, Martin DMA, et al.: Genome sequence of the human malaria parasite Plasmodium falciparum. Nature 2002, 419:498-5II.

25. Gresham D, Ruderfer DM, Pratt SC, Schacherer J, Dunham MJ, Botstein $D$, Kruglyak L: Genome-wide detection of polymorphisms at nucleotide resolution with a single DNA microarray. Science 2006, 3II:1932-1936.

26. Jiang H, Yi M, Mu J, Zhang L, Ivens A, Klimczak L, Huyen Y, Stephens $R$, Su X-z: Detection of genome-wide polymorphisms in the AT-rich Plasmodium falciparum genome using a high-density microarray. BMC Genomics 2008, 9:398.

27. Bolstad BM, Irizarry RA, Astrand M, Speed TP: A comparison of normalization methods for high density oligonucleotide array data based on variance and bias. Bioinformatics 2003, 19: I85-193.

28. Zhou Y, Abagyan R: Match-only integral distribution (MOID) algorithm for high-density oligonucleotide array analysis. BMC Bioinformatics 2002, 3:3.

29. Cowman AF, Galatis D, Thompson JK: Selection for mefloquine resistance in Plasmodium falciparum is linked to amplification of the pfmdrl gene and cross-resistance to halofantrine and quinine. Proc Natl Acad Sci USA 1 994, 9 I: I I 43- I I 47.

30. Ferreira ID, Rosário VE, Cravo PV: Real-time quantitative PCR 
with SYBR Green I detection for estimating copy numbers of nine drug resistance candidate genes in Plasmodium falciparum. Malar J 2006, 5: I.

31. Nair S, Nash D, Sudimack D, Jaidee A, Barends M, Uhlemann A-C, Krishna S, Nosten F, Anderson TJC: Recurrent gene amplification and soft selective sweeps during evolution of multidrug resistance in malaria parasites. Mol Biol Evol 2007, 24:562-573.

32. Carret CK, Horrocks P, Konfortov B, Winzeler E, Qureshi M, Newbold $C$, Ivens A: Microarray-based comparative genomic analyses of the human malaria parasite Plasmodium falciparum using Affymetrix arrays. Mol Biochem Parasitol 2005, I 44: I 77-I86.

33. Nkrumah LJ, Muhle RA, Moura PA, Ghosh P, Hatfull GF, Jacobs WR Jr, Fidock DA: Efficient site-specific integration in Plasmodium falciparum chromosomes mediated by mycobacteriophage Bxb I integrase. Nat Methods 2006, 3:6I5-62I.

34. Jomaa H, Wiesner J, Sanderbrand S, Altincicek B, Weidemeyer C, Hintz M, uuml, rbachova I, Eberl M, Zeidler J, Lichtenthaler HK, Soldati D, Beck E: Inhibitors of the nonmevalonate pathway of isoprenoid biosynthesis as antimalarial drugs. Science 1999, 285: I573-I576.

35. Rohmer M: The discovery of a mevalonate-independent pathway for isoprenoid biosynthesis in bacteria, algae and higher plants. Nat Prod Rep 1999, I 6:565-574.

36. Borrmann S, Adegnika A, Matsiegui P-B, Issifou S, Schindler A, MawiliMboumba D, Baranek T, Wiesner J, Jomaa H, Kremsner P: Fosmidomycin-clindamycin for Plasmodium falciparum infections in African children. J Infect Dis 2004, I 89:901-908.

37. Borrmann S, Issifou S, Esser G, Adegnika A, Ramharter M, Matsiegui P-B, Oyakhirome S, Mawili-Mboumba D, Missinou M, Kun JJ, Jomaa H, Kremsner P: Fosmidomycin-clindamycin for the treatment of Plasmodium falciparum malaria. J Infect Dis 2004, I 90: I 534- I540.

38. Borrmann S, Adegnika AA, Moussavou F, Oyakhirome S, Esser G, Matsiegui P-B, Ramharter M, Lundgren I, Kombila M, Issifou S, Hutchinson D, Wiesner J, Jomaa H, Kremsner PG: Short-course regimens of artesunate-fosmidomycin in treatment of uncomplicated Plasmodium falciparum malaria. Antimicrob Agents Chemother 2005, 49:3749-3754.

39. Cassera MB, Gozzo FC, D'Alexandri FL, Merino EF, del Portillo HA, Peres VJ, Almeida IC, Eberlin MN, Wunderlich G, Wiesner J, Jomaa $\mathrm{H}$, Kimura EA, Katzin AM: The methylerythritol phosphate pathway is functionally active in all intraerythrocytic stages of Plasmodium falciparum. J Biol Chem 2004, 279:5 I 749-5 I 759.

40. Cassera MB, Merino EF, Peres VJ, Kimura EA, Wunderlich G, Katzin AM: Effect of fosmidomycin on metabolic and transcript profiles of the methylerythritol phosphate pathway in Plasmodium falciparum. Mem Inst Oswaldo Cruz 2007, I 02:377-384.

41. Winzeler Lab Website [http://www.scripps.edu/cb/winzeler/soft ware]

42. Ribacke U, Mok BW, Wirta V, Normark J, Lundeberg J, Kironde F, Egwang TG, Nilsson P, Wahlgren M: Genome wide gene amplifications and deletions in Plasmodium falciparum. Mol Biochem Parasitol 2007, I 55:33-44.

43. lafrate AJ, Feuk L, Rivera MN, Listewnik ML, Donahoe PK, Qi Y, Scherer SW, Lee C: Detection of large-scale variation in the human genome. Nat Genet 2004, 36:949-95I.

44. Sebat J, Lakshmi B, Troge J, Alexander J, Young J, Lundin P, Maner S, Massa H, Walker M, Chi M, Navin N, Lucito R, Healy J, Hicks J, Ye K, Reiner A, Gilliam TC, Trask B, Patterson N, Zetterberg A, Wigler M: Large-scale copy number polymorphism in the human genome. Science 2004, 305:525-528.

45. Redon R, Ishikawa S, Fitch KR, Feuk L, Perry GH, Andrews TD, Fiegler $\mathrm{H}$, Shapero MH, Carson AR, Chen W, Cho EK, Dallaire S, Freeman JL, Gonzalez JR, Gratacos M, Huang J, Kalaitzopoulos D, Komura D, MacDonald JR, Marshall CR, Mei R, Montgomery L, Nishimura K Okamura K, Shen F, Somerville MJ, Tchinda J, Valsesia A, Woodwark $C$, Yang F, et al.: Global variation in copy number in the human genome. Nature 2006, 444:444-454.

46. Sebat J: Major changes in our DNA lead to major changes in our thinking. Nat Genet 2007, 39:S3-S5.

47. Ganesan K, Ponmee N, Jiang L, Fowble JW, White J, Kamchonwongpaisan S, Yuthavong Y, Wilairat P, Rathod PK: A genetically hardwired metabolic transcriptome in Plasmodium falciparum fails to mount protective responses to lethal antifolates. PLoS Pathog 2008, 4:el0002I4.

48. Shendure J, Porreca G], Reppas NB, Lin X, McCutcheon JP, Rosenbaum AM, Wang MD, Zhang K, Mitra RD, Church GM: Accurate multiplex polony sequencing of an evolved bacterial genome. Science 2005, 309: I728-I732.
49. Bekal S, Craig J, Hudson M, Niblack T, Domier L, Lambert K: Genomic DNA sequence comparison between two inbred soybean cyst nematode biotypes facilitated by massively parallel 454 micro-bead sequencing. Mol Genet Genomics 2008, 279:535-543.

50. Hillier LW, Marth GT, Quinlan AR, Dooling D, Fewell G, Barnett D, Fox P, Glasscock Jl, Hickenbotham M, Huang W, Magrini VJ, Richt RJ, Sander SN, Stewart DA, Stromberg M, Tsung EF, Wylie T, SchedI T, Wilson RK, Mardis ER: Whole-genome sequencing and variant discovery in C. elegans. Nat Methods 2008, 5:183-188.

51. Trager W, Jenson JB: Cultivation of malarial parasites. Nature 1978, 273:621-622

52. Winzeler EA, Richards DR, Conway AR, Goldstein AL, Kalman S, McCullough MJ, McCusker JH, Stevens DA, Wodicka L, Lockhart DJ, Davis RW: Direct allelic variation scanning of the yeast genome. Science 1998, 28 I: I |94- I | 97

53. Goodyer ID, Taraschi TF: Plasmodium falciparum: a simple, rapid method for detecting parasite clones in microtiter plates. Exp Parasitol 1997, 86: I58-I60.

54. Desjardins RE, Pamplin CL 3rd, von Bredow J, Barry KG, Canfield C]: Kinetics of a new antimalarial, mefloquine. Clin Pharmacol Ther 1979, 26:372-379.

55. Fidock DA, Wellems TE: Transformation with human dihydrofolate reductase renders malaria parasites insensitive to WR992 IO but does not affect the intrinsic activity of proguanil. Proc Natl Acad Sci USA 1997, 94:10931-10936.

56. PlasmoDB: The Plasmodium Genome Resource [http:// www.plasmodb.org] 